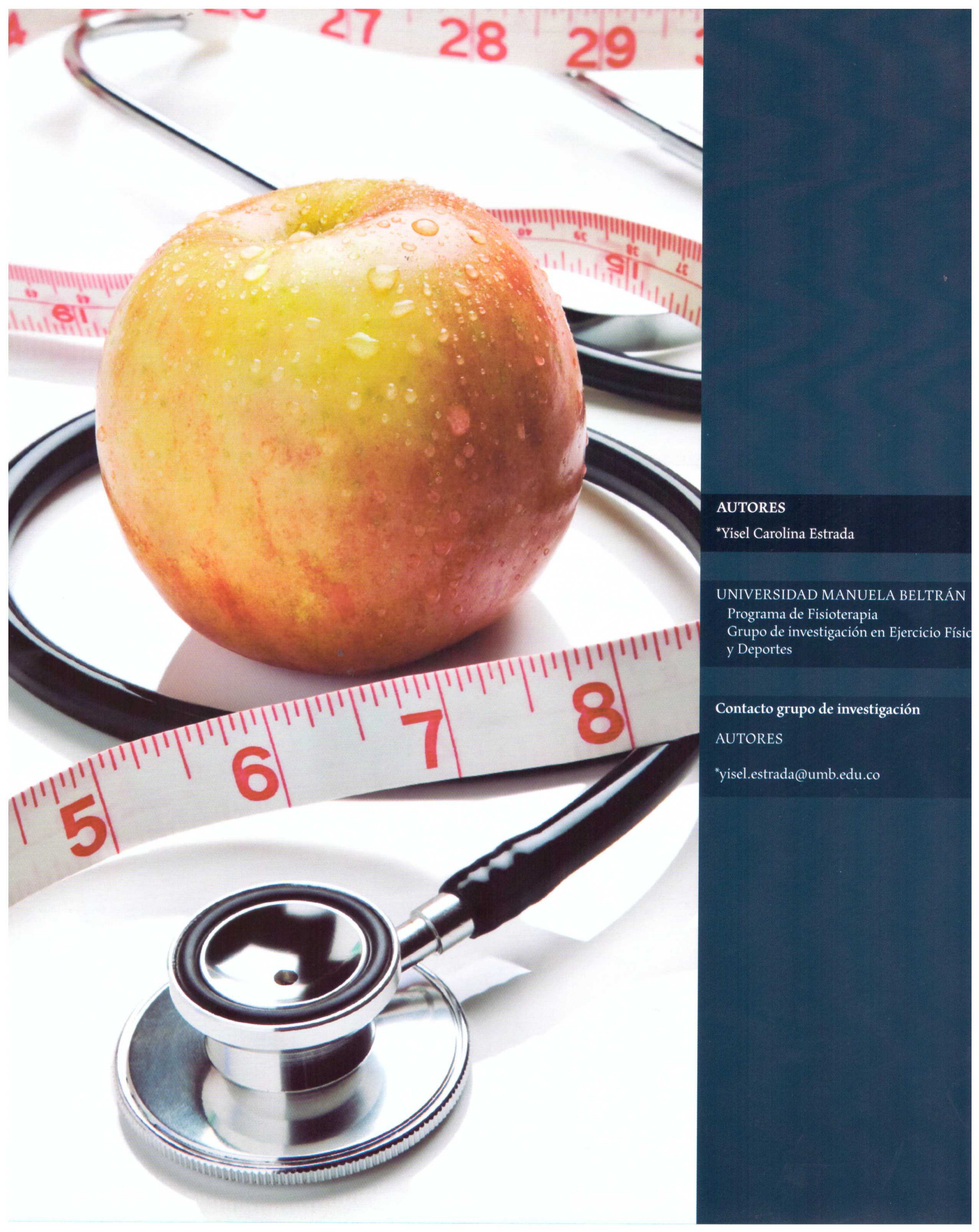




\section{REVISIÓN SISTEMÁTICA SOBRE LAS BATERIAS DE EVALUACIÓN USADAS EN EL EXAMEN DE LA CONDICIÓN FÍSICA}

Fecha de recepción Abril de 2010 . Fecha de Aprobación 10 de Junio de 2010

\section{RESUMEN}

Se realizó una revisión sistemática, en la cual se determinaron las baterías de medición que se usan para examinar la Condición Física por medio de la valoración de las diferentes Cualidades Físicas que hacen parte de la misma. En este artículo también se determinaron los principales usos de las baterías de medición mayormente utilizadas, teniendo en cuenta variables tales como: género, edad y grupos etáreos, en los cuales son empleadas. También se tuvieron en cuenta los países en los que se han realizado investigaciones haciendo aplicación de alguna de las baterías de exámen de la Condición Física. Todos los artículos tenidos en cuenta en esta revisión sistemática, tuvieron en cuenta investigaciones realizadas en Latinoamérica, Norteamérica y Europa, en los últimos 20 años, con la participación de investigaciones que tuvieran diseños descriptivos longitudinales y transversales, así como también se tuvieron en cuenta investigaciones de diseño experimental. Se puede concluir que existe actualmente una variedad limitada de baterías que son comúnmente utilizadas para la medición y evaluación de la Condición Física, entre las que se destaca la Batería EUROFIT. Se concluye de igual forma, que hace falta realizar más investigaciones que identifiquen los usos y aplicaciones de la batería de evaluación anteriormente nombrada, teniendo en cuenta que esta herramienta es una de las más adecuadas para examinar con suficiente validez y confiabilidad la Condición Física.

PALABRAS CLAVE

Actividad Física, Aptitud Física, Capacidades Físicas Coordinativas, Capacidades Físicas Condicionales, Condición Física.

\section{ABSTRACT}

We conducted a systematic review of the measurement batteries used to evaluate Fitness through the assessment of its different physical qualities. In this paper we determined the main applications of the most used measurement batteries, considering variables such a gender, and age groups to whom they are applied. We considered too the countries where some research had been conducted applying any of the Fitness measuring batteries. All of the reviewed papers included research in Latin America, North America and Europe during the last 20 years, with longitudinal and transversal descriptive design as well as experimental design. It can be concluded that currently exists a limited range of batteries that are commonly used for the measurement and evaluation of Physical Fitness, among them prevailing the use of the EUROFIT battery. We concluded too that more research is needed to identify the uses and applications of the previously mentioned assessment battery, bearing in mind that this tool is one of the most appropriate to examine Physical Fitness with enough validity and reliability. 
$\mathrm{E}$ concepto de Condición Física se introdujo en un principio con un enfoque más marcado hacia el campo de la Educación Física, a principios del siglo XX (más exactamente en 1916). Desde este campo del conocimiento se define la Condición Física como "la capacidad de realizar trabajo diario, con vigor y efectividad, con máxima eficiencia y mínimo gasto energético, retardando la aparición de fatiga y previniendo la aparición de lesiones" (1). Parte de la forma como se define la Condición Física desde la Educación Física se entrelaza con lo que se concibe en la Salud para la Aptitud Física, de manera que para Navarro et al (1997), la Condición Física "es parte de la condición total del ser humano y comprende muchos componentes, cada uno de los cuales es específico en su naturaleza" (2). La Condición Física, también denominada como Aptitud Física, supone la combinación de diversos aspectos en diversos grados. La resistencia aeróbica y la resistencia muscular, junto con la fuerza y la flexibilidad, pueden considerarse como aspectos de la salud relacionados con la Condición o la Aptitud Física (2).

La Condición Física es definida como "la capacidad que tiene el cuerpo para moverse en forma óptima a partir de un conjunto de capacidades, factores o cualidades motrices que tiene cada individuo, y que, mediante su desarrollo conforma la base de la Condición Física de la persona" $(2,3)$.

Teniendo en cuenta lo anterior, se concluye que la Condición Física es el conjunto de aspectos que a nivel físico permiten que el individuo se mueva, gastando el mínimo de energía. Esta definición se toma muy apegada a la definición que tiene en inglés el término Physical Fitness, recogiendo también la posibilidad de mejorar las capacidades del aparato locomotor para desempeñarse mejor en cuanto al movimiento se refiere $(3,4,5)$.

De igual forma, complementando lo anteriormente expuesto (4), el concepto de Condición Física puede ser representado por un triángulo, cuyos lados corresponden a los tres componentes principales de la misma: orgánico, motriz y cultural.

La dimensión orgánica se refiere a los procesos de producción de energía y al rendimiento. Es el más directamente relacionado con la salud. Para valorar esta dimensión orgánica, se pueden realizar los test de resistencia cardio respiratoria, como el de Cooper o el de Luc - Leger. Estas pruebas muestran un alto grado de validez en edades escolares y en adultos (4).

En la dimensión motriz se ponen en marcha las capacidades psicomotrices necesarias para concebir el componente motriz de desarrollo de cualquier individuo: cualidades físicas tales como fuerza, resistencia muscular y velocidad. La batería de evaluación EUROFIT, por ejemplo, mide la aptitud motriz general, pero no el nivel de habilidad técnica en un deporte dado o en un movimiento específico (2).
Por último, la dimensión cultural, constituve el tercer lado , triángulo de la Condición Física y refleja la influencia de los nive de Condición Física $(2,3)$.

La motricidad humana, vista desde el concepto de la Aptit Física, se puede estudiar desde los puntos de vista coordinativc condicional. Dentro de lo coordinativo se tienen en cuenta capa dades como la diferenciación, acoplamiento, orientación, cambio ritmo, relajación y velocidad (5).

Los individuos poseen capacidades físicas condicionales $\mathrm{q}$ se pueden desarrollar y mantener en forma limitada de acuerdo factores tales como el genético, estructural, fisiológico, biomecánic psicológico y energético. Las capacidades físicas que pertenecen esta clasificación son: la fuerza, la resistencia, la velocidad, la rap dez y la flexibilidad (5).

Todas las capacidades físicas en mayor o en menor medida se e: cuentran presentes en el desarrollo del movimiento corporal hum no, en cualquier ejercicio físico o deporte, así como en el desarrol de praxias cotidianas como la marcha o la carrera, entre otras (6).

Las capacidades físicas condicionales y coordinativas son las qu determinan la Condición Física del individuo. La Aptitud Físic de un individuo entonces depende de la capacidad o fuerza a niv muscular, de la resistencia para mantener dicha fuerza en el tiempr y en tercer lugar de la capacidad del corazón y los pulmones par aportar energía al sistema músculo esquelético. El hecho de tene una adecuada Condición Física y una buena salud es imprescindibl para que el ser humano se pueda adaptar a la vida cotidiana y par que, a partir de la misma, pueda aprender praxias motrices má complejas, bajo ciertas normas, para el desarrollo del ejercicio físic o de un deporte en particular. En el momento en que las cualidade físicas que componen la Condición Física se vislumbran desde un perspectiva deportiva, se habla más de una Aptitud Física, en e sentido de aprovechar dichas capacidades físicas para la realizació de una práctica deportiva $(7,8,9,10,11,12)$.

\section{Relación entre actividad física y condición física}

La actividad física es un elemento vital tan complejo, que st medición y evaluación tiende a ser complicada y difícil, dada la importancia para el ser humano (13). Uno de los elementos que per. miten realizar una medición bastante cercana de la actividad física es la evaluación de la Condición Física, la cual permite conocer e] estado inicial del individuo, teniendo así una visión general de la aptitud física de la persona que va a realizar algún tipo de actividad física. Generalmente, la evaluación pre realización de actividad física en cualquiera de sus variantes (Ejercicio Físico o Deporte), exige del equipo preparador o atendiente, de la evaluación concreta de la Condición Física, haciendo especial énfasis en aquellas cualidades 
que determinan el desarrollo de adecuados procesos de actividad ejercicio Físico y/o Deporte (12).

De acuerdo con lo anterior, las capacidades o cualidades físicas que intervienen en el desarrollo de movimiento expresado en el Ejercicio Físico o en el Deporte, que se deben medir y evaluar en cada individuo, y que se espera mejorar con la realización de cualquier tipo de Actividad Física son las siguientes:

- Velocidad (la cual se puede clasificar como una capacidad física mixta, es decir tiene tanto de capacidad física coordinativa como de capacidad física condicional) (13).

- Flexibilidad (la cual se clasifica como capacidad física condicional) (13).

- Fuerza (la cual se clasifica como capacidad física condicional).

- Resistencia aeróbica: (que se clasifica como una capacidad física condicional) (14).

Las anteriores cualidades físicas pueden ser medidas por diversos test o baterías ya existentes creadas para tal fin. Aunque la valoración de la Condición Física ha sido un aspecto que siempre ha preocupado al ser humano, no ha sido sino hasta el siglo pasado, cuando han aparecido los primeros trabajos con un carácter científico, en los cuales se han realizado procesos de medición y evaluación de la Condición Física, a través de la medición y evaluación de las cualidades físicas (condicionales y coordinativas) (14).

Según lo reportado por García Manso et al (6), se ha observado la existencia de métodos de medición del rendimiento motor, en los que se hace uso de metodologías que solo tienen en cuenta elementos antropométricos sin aplicaciones metodológicas sístemáticas en cuanto a la parte de las cualidades físicas como tałes $(15,16)$.

Sin embargo, en los últimos 50 años se ha observado la evolución y la maduración por el interés en las baterías de análisis del nivel de Condición Física, así como por la interpretación de datos obtenidos de estas (16). Los primeros protocolos estaban limitados a valorar la Condición Física desde el punto de vista motriz, y la interpretación de los resultados estaba limitada a su vez a realizar comparaciones con valores percentiles (16).

El mayor giro producido en el interés por la valoración del nivel de Condición Física se produjo cuando los investigadores establecieron diferencias entre la Condición Física relacionada con el Rendimiento Deportivo de un individuo, con la Condición Física relacionada con el estado de salud, basándose la definición de Condición Física para este caso concreto en la relación Condición Física - Salud realización de Actividad Física (17). Los fenómenos de integración salud - actividad física - Condición Física se consolidaron concretamente en la década de los 50, como una tendencia a nivel mundial. Así, desde este momento, la mayoría de las baterías y protocolos que existen para valorar el nivel de la Condición Física se orientaron hacia los intereses de la población general, produciéndose el mismo efecto en el interés de los investigadores, el cual se volcó claramente hacia este campo (17).
De igual forma, y de acuerdo con lo anterior, en la década de los 50, más exactamente en 1958, aparece una de las baterías de evaluación de la Condición Física, generada en este caso por la Asociación Americana para la Salud, la Educación Física, la Recreación y la Danza, la cual tiene en cuenta siete pruebas con escalas de percentiles para cada una de las cualidades físicas, en función de la edad (17). Esta batería de evolución recibió el nombre de AAPHERD. En 1976 sufrió ajustes en su estructura y quedó conformada por seis pruebas, que son las siguientes:

- Prueba de tracción de brazos (la cual mide la resistencia muscular de los miembros Superiores - MMSS).

- Prueba de abdominales con piernas flexionadas (la cual mide la resistencia muscular de tronco).

- Prueba de carrera de ida y vuelta sobre 10 yardas (la cual mide la agilidad).

- Prueba de salto largo con pies juntos (que sirve para medir la fuerza explosiva en el tren inferior).

- Prueba de velocidad sobre 50 yardas (para la medición de la velocidad).

- Prueba de resistencia cardiovascular con varias posibilidades: recorrer 600 yardas en 9 minutos de carrera o en 12 minutos de paso rápido(para la medición y evaluación de la resistencia cardiovascular) (18).

Hacia 1980 se realiza una variación de la batería AAPHERD, que se denomina Health Releated Fitness Test, compuesta por cuatro pruebas:

1. Correr una milla o 9 minutos.

2. Sumatoria de los pliegues cutáneos del tríceps y del subescapular (para la medición de la composición corporal del individuo).

3. Abdominales de un minuto (para la evaluación de la resistencia muscular de tronco).

4. Test de Wells y Dillon o "sit and reach" (para la medición y evaluación de la flexibilidad) (18).

En el año de 1978, en la ciudad de París, nació una de las baterías de evaluación de la Condición Física más utilizadas actualmente para verificar y conocer el estado inicial de la Aptitud Física tanto de las personas que se encaminarán a la realización de actividad física o se enfocarán más a la realización de Ejercicio Físico o Deporte. Por su lugar de origen esta batería de evaluación recibió su nombre de Test EUROFIT, lo cual hace referencia a la evaluación del Fitness de las personas habitantes de Europa, pertenecientes a los distintos grupos etáreos $(19,20)$.

En el momento en que nacía la batería EUROFIT, se advertía una gran preocupación por la Condición Física presentada en ciertos grupos etáreos, como la población infantil, en el seno de los países miembros de la comisión que creó dicha batería. La preocupación acerca del estado de la Condición Física se debía a que después de la segunda guerra mundial se aumentó el uso de elementos tales como el carro y la televisión, lo cual condujo al aumento del sedentarismo en algunos sectores de la población (20). 
El proyecto de creación de la batería de evaluación de la Condición Física EUROFIT también nació con el ánimo de aplicar el principio del deporte para todos, de acuerdo con las directrices del consejo de Europa, con el propósito de que todos los ciudadanos europeos pudieran conocer la satisfacción que aporta la realización de Actividad Física favorecedora del desarrollo (20).

La batería EUROFIT reúne una serie de tests de evaluación de la Aptitud Física que guardan estrecha relación con el concepto de salud, y que guardan el siguiente orden:

- Tests de prioridad 1: presentan una relación muy estrecha y contrastada con la salud, abarcando la resistencia aeróbica, la resistencia muscular del tronco y la flexibilidad.

- Tests de prioridad 2: los cuales miden la potencia de miembros superiores e inferiores, cuyas relaciones entre salud y Condición Física son menos evidentes.

- Tests de prioridad 3: que incluyen la dinamometría manual y el golpe de placas y que además de dar indicaciones más específicas en materia de salud, miden aptitudes importantes para ciertos grupos especiales (2). La batería EUROFIT para la valoración de la Condición Física mide las cualidades Físicas Condicionales por medio de las siguientes pruebas:

- Fuerza máxima, a través dela dinamometría de miembros superiores.

- Fuerza explosiva, mediante la prueba de salto largo sin carrera de impulso (o salto a pie junto).

- Tiempo de reacción simple, a través de la prueba del bastón de Galtón.

- Rapidez de manos, a través de lasprueba de tapping.

- Rapidez de pies, a través de la prueba de Skipping.

- Aceleración, mediante la prueba de carrera de 20 o 30 metros lanzada con 10 a 20 metros de impulso.

- Velocidad, a través de la carrera de 20 o 30 metros.

- Flexibilidad, a través del test de Wells y Dillon o test de "sit and reach".

- Resistencia aeróbica, a través de el test de Luc Leger o course - navette.

- Consumo Máximo de Oxígeno a través del test de Luc - Leger.

Ya que esta batería evalúa las cualidades físicas condicionales, y teniendo en cuenta lo dicho por Ramos et al, "las capacidades físicas condicionales son las que determinan el desarrollo motriz, así como la Condición Física del individuo", se constituye entonces en uno de los métodos de evaluación de la Aptitud Física más completos que hasta ahora existen para tal fin (3). Esta batería es ampliamente utilizada en los países de la Unión Europea, para la evaluación y análisis de la Condición Física en personas potencialmente sanas, o en personas con alguna capacidad física excepcional que desarrolle algún tipo de Ejercicio Físico o de Deporte (21).

\section{METODOLOGÍA}

La búsqueda y recolección de los diferentes estudios se llevó a cabo mediante la revisión de revistas indexadas relacionadas con e tema. También se tuvo en cuenta la revisión de bases de datos par: la realización de la búsqueda de los artículos que hacen parte de esti revisión. Los estudios analizados fueron seleccionados de acuerdo cor los siguientes criterios:

- Revisiones documentales de los últimos 20 años (de 1988 a 2009)

- Estudios Descriptivos de tipo transversal y longitudinal (tantc prospectivo como retrospectivo).

- Estudios realizados en poblaciones europeas, latinoamericanas) norteamericanas.

- Estudios publicados en bases de datos o en revistas indexadas.

- Estudios realizados en poblaciones etáreas: níños de 7 a 11 años jóvenes de 12 a 17 , adultos jóvenes de 20 a 60 años y adulto: mayores de 60 a 90.

La búsqueda de los artículos que hacen parte de este estudio, y que fueron utilizados para la realización de esta revisión sistemática se efetuó en bases de datos tales como PubMed Central, HINARI HAPI y PEDro. PubMed es un archivo de investigación biomédice y de revistas de ciencias de la vida que cuenta con más de 1.500 .00 c artículos, de más de 450 revistas electrónicas. PEDro es la Base de Datos de Fisioterapia Basada en la Evidencia que incluye reseñas bi. bliográficas, resúmenes de ensayos clínicos controlados y revisiones sistemáticas en Fisioterapia.

HINARI fue establecida por la OMS, como una base de datos que contiene una de las más extensas colecciones de literatura biomédica y de salud, con fácil acceso a los países en vías de desarrollo. HAPI así mismo, pone a disposición de los investigadores en Latinoamérica, más de 275,00o publicaciones relacionadas con temas de salud, ciencias naturales y sociales.Los términos clave empleados en la búsqueda de los artículos que hacen parte de esta revisión sistemática fueron:

- Physical fitnness

- Physical fitness evaluation.

- EUROFIT evaluation battery

- Evaluation/baseline.

- Physical skills.

Los datos extraídos de los artículos incluyen el tamaño muestral, el tipo de estudio, el periodo de tiempo en que se aplicaron las intervenciones y las evaluaciones, las baterías aplicadas para la evaluación y medición de la Condición Física, los indicadores (tests y medidas) de los resultados y los resultados más relevantes obtenidos en cada estudio.

\section{RESULTADOS}

En la realización de la búsqueda en las bases de datos electrónicas, se encontraron 76 referencias, de las cuales 50 cumplieron con los criterios de selección y se incluyeron en la revisión.

Dada la importancia que ha cobrado la evaluación de la Condición Física tanto en población potencialmente sana como en 
población que realiza Ejercicio Físico o Deporte, se considera que los procesos de evaluación de la Aptitud Física mediante la aplicación de los distintos métodos evaluativos, son importantes por que permiten conocer el estado inicial del individuo sometido a evaluación, desde la dimensión motriz, a través de la valoración de aspectos tales como resistencia aeróbica, resistencia muscular de tronco y extremidades, velocidad y flexibilidad entre otros, aspectos clave para el desarrollo de una adecuada condición de salud. De acuerdo con la revisión sistemática realizada en el presente artículo, se observa que los países que en los últimos 20 años han realizado más estudios para la determinación de la Condición Física se encuentran en Europa. El $56 \%$ de los estudios revisados (que corresponde a 28 de los 50 artículos revisados) se han hecho en España, mientras que el 20\% (10 de 50) se ha realizado en el Reino Unido (UK). Se observa también a partir de la revisión de estudios realizados, que los Estados Unidos de América son responsables del $8 \%$ (4 de 50) de los estudios realizados, seguidos por Argentina (4\%), México (2\%) y Colombia (2\%). (Ver figura 1).

De igual forma, para el caso concreto de esta revisión sistemática, se ha encontrado que los estudios analizados, se enfocan en evaluar y valorar la Condición Física en ambos géneros (Hombres y Mujeres), teniendo en cuenta diferentes grupos etáreos (niños, adolescentes, adultos jóvenes, adulto mayor y tercera edad). Se observa que la mayoría de los estudios de Condición y/o Aptitud Física se realizaron en diferentes grupos etáeros del género masculino (86\%, o sea 43 de los 50 artículos). Le siguen los estudios que tienen en cuenta la evaluación de la Condición Física para los dos géneros (hombres y mujeres), en los distintos grupos etáreos $(14 \%$ de los artículos analizados en esta revisión, correspondientes a 7 de 50 artículos tenidos en cuenta para la realización de esta revisión, fueron hechos teniendo en cuenta ambos géneros). Dentro de la revisión de estudios que se realizó, no se encontró ningún estudio que tuviera en cuenta la valoración de la Condición Física únicamente para el género femenino (ver figura 2).
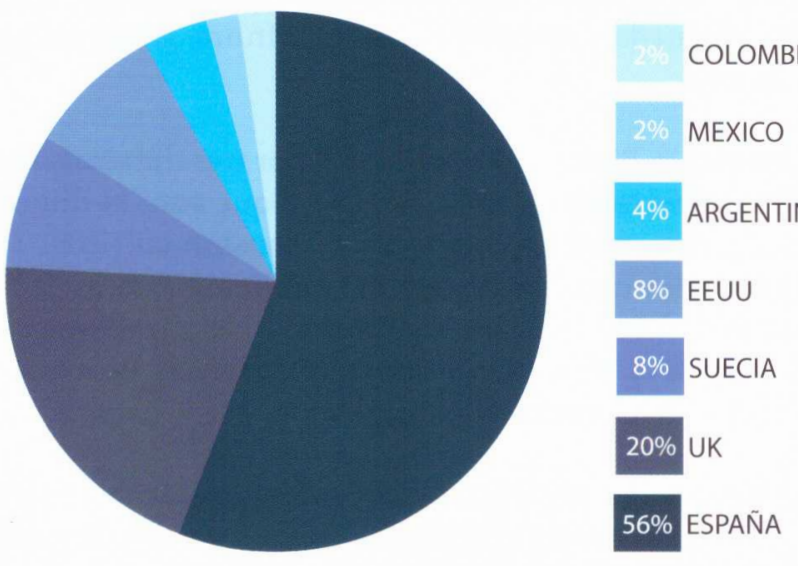

Figura 1. Porcentaje de artículos publicados por países. Fuente. El autor, 2009.

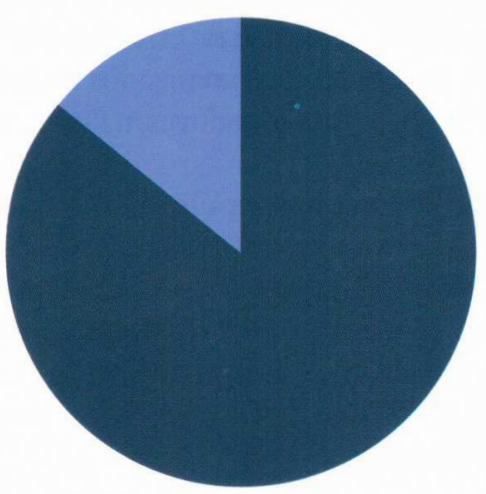

Figura 2. Porcentaje de estudios analizados en esta revisión sistemática asociados con la variable del género. Fuente: el autor, 2009

Teniendo en cuenta los resultados obtenídos en cuanto al género se refiere, se observa que los grupos etáreos valorados mediante los distintos test de medición de la Condición Física fueron en su orden adultos jóvenes y niños ( $15 \%$ en adultos jóvenes, correspondiente a 8 de 50 artículos revisados) y $13 \%$ en población infantil, correspondiente a 7 de 50 artículos revisados), seguido por la realización de valoraciones de la Condición Física en adolescentes, adulto mayor, poblaciones de Deportistas y en la determinación y/o seguimiento de estados de enfermedad o de lesión (ver figura 3 ).

De igual forma, se hizo una revisión sistemática por año de publicación de los artículos. El año en que más se publicaron estudios sobre cómo medir y evaluar la Condición Física fue 1990 (22\%, o sea 11 de los 50 artículos revisados). Le siguen 1994 y 1997 respectivamente (ver figura 4 ).

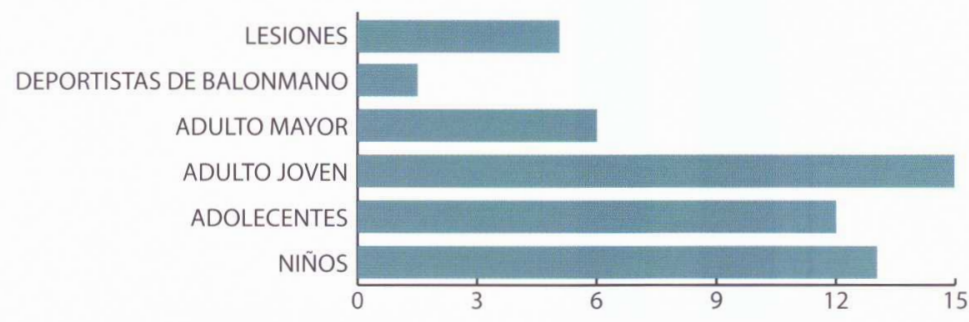

Figura 3. Porcentaje de estudios publicados que realizan valoración de la Condición Física por grupos etáreos. Fuente: el autor, 2009.

Uno de los objetivos fundamentales de esta revisión sistemática es determinar cuales son las baterías de evaluación existentes que se encuentren enfocadas en la evaluación y valoración de la Aptitud Física. De acuerdo con la revisión de estudios que se realizó, se encontró que las baterías de evaluación de la Condición Física más comúnmente usadas son: EUROFIT test, el test de evaluación de la Condición Física de la AAPHERD, el test de FLEISMAN, el test de CAHPER y el test de la ICSPFT. Entre los estudios revisados se encontró que un 96\% (48 de los 
50 artículos revisados), se hicieron bajo las directrices del test EUROFIT, en tanto que solo el 4\% ( 2 de los estudios) se realizó con el test de la AAPHERD. A pesar de la existencia de otras baterías de evaluación de la Condición Física, en los estudios analizados no se encontró el uso o la aplicación de dichas baterías de evaluación (Ver figura 5).

Al hablar de los test existentes para la valoración de la Condición Física, en los artículos analizados en esta revisión sistemática, se observa que se prefiere el uso de la batería EUROFIT por encima de las demás, con lo cual se puede afirmar que, actualmente, esta batería de evaluación es la más utilizada para la evaluación de la Aptitud Física, teniendo en cuenta los componentes de la Condición Física como tal.

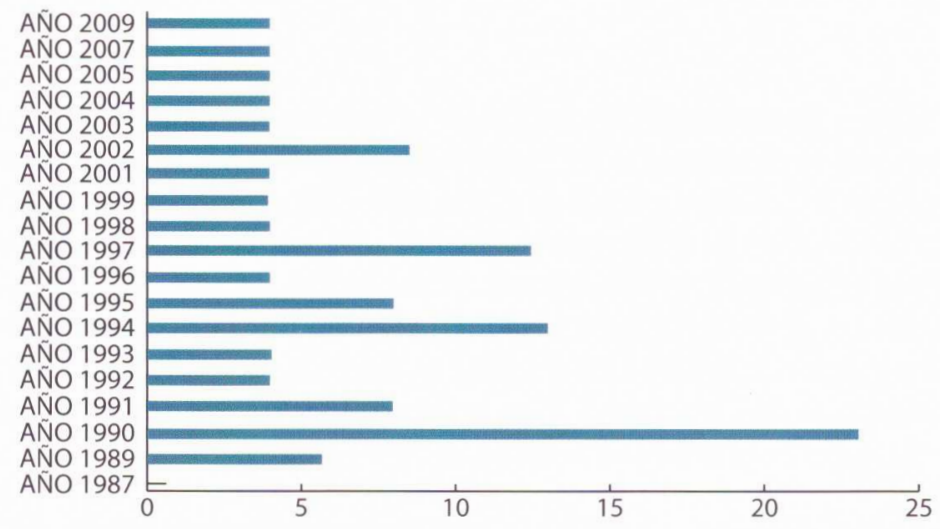

Figura 4. Porcentaje de Estudios publicados por año, según criterios de inclusión. Fuente: el autor, 2009.

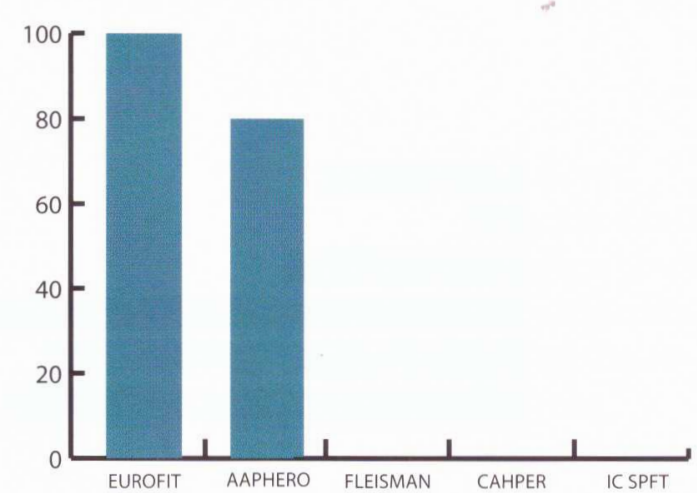

Figura 5. Porcentaje de Test de evaluación de la Condición Física, según los estudios analizados en este artículo. Fuente; el autor, 2009

En cuanto a los criterios evaluados por las baterías de valoración de la Condición Física, se observó en esta revisión sistemática que fueron las siguientes: la velocidad, la fuerza explosiva, la fuerza máxima, la flexibilidad y la resistencia aeróbica. También se evaluaron aspectos tales como la resistencia muscular en tronco y en extremidades, pero estos últimos en menor medida (ver figura 6).

\section{CONCLUSIONES}

El ser humano tiene cuerpo y capacidad para moverse. Estc implica que hay un conjunto de factores, capacidades o cualidades que tiene cada individuo, y que, mediante su desarrollo, a través de la realización de algún tipo de Actividad Física, Ejercicio Físicc o Deporte, conforman la Condición Física de la persona, tambiér denominada como Aptitud Física (22).

Atendiendo al significado del lenguaje, conceptos tales comc el de Condición Física, Aptitud Física, forma física, preparación física, eficiencia motriz, capacidad motriz, etc, son términos quє de forma más coloquial se emplean indiferentemente para designar una misma realidad: la necesidad de realizar procesos de evaluación y valoración de la Condición Física, teniendo en cuenta tanto la definición del concepto así como los componentes del mismo.

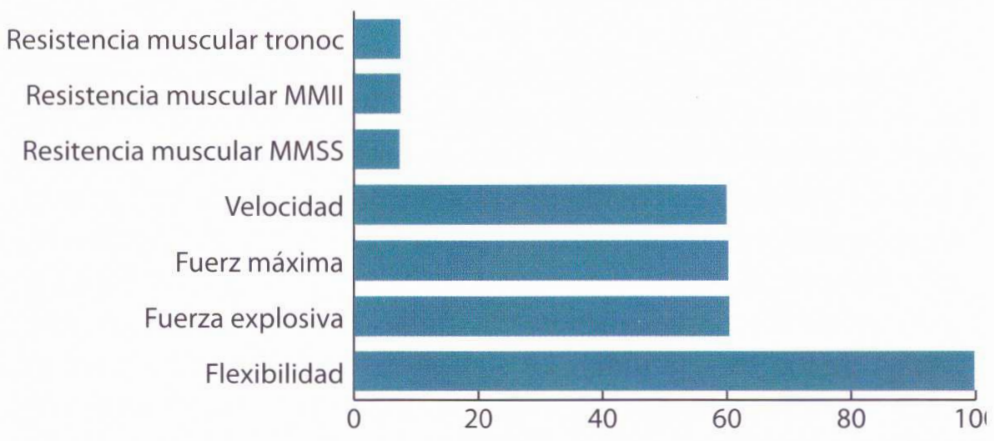

Figura 6. Porcentaje de variables medidas y valoradas de acuerdo al test o batería utilizada. Fuente: el autor, 2009.

El concepto de Condición Física es el que actualmente se ajusta a los propósitos de revisión de este artículo, además de ser aceptado como el término más reconocido para definir el conjunto de capacidades o de cualidades que permiten moverse de forma adecuada y armónica, con el mínimo gasto energético $(23,24,25,26)$.

La Condición Física está compuesta por tres dimensiones que se pueden representar en un triángulo y que son: la dimensión orgánica, la dimensión motriz y la dimensión cultural. De las tres dimensiones que conforman la Condición Física, es la primera que se nombró (Dimensión Orgánica) la que se evalúa más específicamente a través de las variadas baterías de evaluación reconocidas a nivel mundial $(26,27,28)$.

En la dimensión orgánica de la Condición Física se tiene en cuenta, para términos de evaluación y de valoración, la medición de las capacidades físicas condicionales tales como la Flexibilidad, la Fuerza Explosiva, la Fuerza Máxima, la Velocidad y la Resistencia Aeróbica. Esta batería de evaluación de la Condición Física también tiene en cuenta la valoración de las características 
antropométricas tales como talla, peso, índice de masa Corporal (IMC), porcentaje de masa magra y porcentaje de masa grasa, haciendo este método de evaluación más completo aún. La razón de evaluar las capacidades físicas condicionales y las características antropométricas de los sujetos se respalda en el hecho de que dichas cualidades son las que determinan el desarrollo físico del movimiento corporal humano $(28,29,30)$. De forma indirecta, al evaluar todos los componentes de la Dimensión Orgánica de la Condición Física, se pueden obtener resultados que permitan la evaluación indirecta de la Condición Física completa.

Dados los resultados obtenidos por la revisión analítica de los estudios, se observa que de las baterías de evaluación existentes para evaluar la Condición Física, la que reúne todos los requisitos para una adecuada evaluación y valoración de la dimensión orgánica, es el test EUROFIT, el cual dentro de sus componentes tiene en cuenta las cualidades físicas condicionales como el respaldo o cimiento de la Condición Física en general $(31,32,33,34)$. Por esta razón, así como por su contenido y validez, el test EUROFIT es actualmente una de las herramientas de más frecuente uso para la determinación y conocimiento de la Condición Física en distintos tipos de población con necesidades específicas. Por lo tanto es de esperarse que los resultados obtenidos en esta revisión sistemática sean los que se obtuvieron.

La razón por la cual se encontraron más estudios con la aplicación de la batería EUROFIT para la valoración de la Condición Física, es que a mediados de 1990 se desarrolló en la ciudad de Izmir en Turquía, el congreso europeo con respecto a la batería EUROFIT para la valoración de la Condición Física. En este congreso se presentaron numerosos trabajos que utilizaban la batería como herramienta para la evaluación de la Condición Física en varios tipos de población, tomando ambos géneros y diferentes grupos etáreos. $(35,36,37,38)$.

Dado que los resultados de la aplicación de la batería de evaluación EUROFIT permitieron realizar aproximaciones bastante cercanas de la situación de la Condición Física, teniendo en cuenta las características tan distintas de la población Europea así como los distintos usos para los cuales se aplicó esta batería de evaluación, y dados los buenos resultados obtenidos, se decidió en ese congreso reconocer la batería de evaluación y valoración de la Aptitud Física como una herramienta primordial para la evaluación inicial de la Condición Física del individuo, bien sea en condiciones de optimización de la forma física, o bien sea en un ámbito clínico $(39,40,41,42,43,44)$. Lo anterior corrobora que el test de evaluación de la Condición Física de EUROFIT es propicio por sus fundamentos, para la evaluación de diversos tipos de población en distintas condiciones de salud y enfermedad.

Se observó en los resultados obtenidos en esta revisión sistemática que la batería de Evaluación de la Condición Física EUROFIT, ha sido empleada para la valoración de la Condición
Física en diversos grupos etáreos, siendo más prevalente su uso en población de adolescentes y en población infantil menor de 12 años, en edad escolar. Este uso tan marcado para este tipo de grupos etáreos denota que la batería EUROFIT está diseñada de tal manera que no sólo permite medir los componentes condicionales de la Condición Física, sino que también puede valorar estos mismos, permitiendo establecer correlaciones entre las tres dimensiones que conforman la Aptitud Física antes mencionadas. Así mismo, si bien el origen de la batería de evaluación de la Condición Física de EUROFIT tuvo otras causas, también fundamentó su creación en la necesidad de la evaluación de poblaciones tales como la infantil o como la de adolescentes, dando una respuesta a la necesidad de los procesos de caracterización y conocimiento general de dichos grupos etáreos, por lo menos a lo que al estado físico se refiere, para luego poder implementar en ellos, alternativas de mejoramiento de su Aptitud Física (). A pesar de que en ese momento se generó esta necesidad, actualmente se siguen planteando preguntas acerca del estado físico de niños y adolescentes, para implementar en ellos planes de acción y mejoramiento del mismo, a través de la realización de diversos tipos de actividad física; y es claro que estos planteamientos se consideran como problemáticas actuales de salud pública $(46,47,48,49,50,51,52)$.

Se observa con gran sorpresa que la batería de evaluación de la Condición Física EUROFIT, ha comenzado a ser aplicada en poblaciones diferentes a aquellas para las que fue creada. Este es el caso de los estudios que se han encontrado en países como Colombia, México y Argentina, entre otros, que ponen en evidencia la necesidad de realizar procesos de reconocimiento de las características de la condición de salud más comunes en los diferentes grupos etáreos, siguiendo la misma línea de trabajo con la cual se creó la batería EUROFIT.

Sin embargo es necesario seguir realizando estudios de caracterización de la Condición Física en Latinoamérica, ya que los resultados obtenidos pueden ser muy útiles para establecer programas y alternativas concretas encaminadas al mantenimiento o mejoramiento de la Condición Física, a partir del conocimiento profundo de las características de la población latinoamericana. Del mismo modo, la aplicación de la batería EUROFIT en estudios que se realicen en Latinoamérica permitirá hacer adecuados seguimientos a los programas y alternativas de mejoramiento o mantenimiento de la Condición Física y el estado de salud.

Se hace necesaria una revisión más exhaustiva de las aplicaciones que puede tener el test EUROFIT, en cuanto a la evaluación y valoración de la Condición Física en poblaciones en donde su uso es mínimo, como por ejemplo en Latinoamérica. También es necesario destacar y dar a conocer todos los posibles usos para los cuales este test ha sido creado. Es útil también dar a conocer este test a la comunidad de fisioterapeutas en Colombia y en Latinoamérica, para que se difunda su uso como herramienta útil para la evaluación realizada en el quehacer de la Fisioterapia. 
1. CLARKE. (1967) Definiciones de condición física. Ed Mc Graw Hill. USA.

2. Navarro Valdivieso et al. (1997). La condición física en la población adulta de la Gran Canaria, y su relación con determinadas actitudes y hábitos de vida. Tesís doctoral. Universidad de Las Palmas - Gran Canaria.

3. Ramos et al (2007). Evaluación antropométrica y motriz condicional en niños de 7 a 18 años en edad escolar. Editorial Universidad de Caldas. Manizales, Caldas. Cap 4.

4. Mahoney et al. (1990). Application of EUROFIT Test to various test of physical fitness. CDDS - CE.

5. Gamble et al. (1990). Application of EUROFIT Test to various test of physical fitness. CDDS - CE.

6. García Manso et al. (2005). Pruebas para la valoración de la capacidad motriz en el deporte. Evaluación de la condición física. Editorial Gymnos, Madrid, España. Cap 1.

7. Boreham et al. (1990). Application of EUROFIT Test to various test of physical fitness. CDDS - CE.

8. González, Redondo et al (2007). Evaluación de la Condición Física escolar iun fin o un medio?. Universidad de Zaragoza Pg 309-313.

9. Oja et al. (1990). Evaluación de la condición física para la población española en relación con la salud. CDDS - CE.

10. Murphy et al. (1990). Application of EUROFIT Test to various test of physical fitness. CDDS - CE.

11. Tuxworth et al. (1990). Evaluación de la condición física para la población española en relación con la salud. CDDS - CE.

12. Renson et al. (1990). Tracking and prediction of adult fitness in males between th ages of 13 and 30 years. CDDS - CE.

13. Cepero Rojas et al. (1997). Intervención didáctica global sobre el proceso de enseñanza - aprendizaje deportivo en la asignatura de educación física en el contexto de la LOGSE. Grupo editorial Universitario LOGSE. $(371-386)$.

14. Jiménez et al (2007). La valoración de la aptitud Físíca y su relación con la salud. Journal of Human Sport and Excersice. Vol II, N II. Pg 53-71.

15. Mendoza, N (2007). Condición Física, habilidades deportivas y calidad de vida. Ed ministerio de educación y ciencia. Madrid, España. Página 4-12.
16. Casterad et al (2004). La medición de la condición física saludable: aplicación de la batería EUROFIT en adultos. Revista Digital EF DEPORTES. Año 10, Número 68.

17. Dennison Barbara et al. (1988). Chilhood physical fitness test: predictor of adult physical activity levels. Pediatrics. 82: 324 - 330.

18. Nogueira Rojo et al (2002). Valoración de la condición física en niños de 11 a 12 años de edad con distintos niveles socioeconómicos. Rev. Int. Med. cienc. fis. aplic. Volumen 2, número 6.

19. Fernández Carmina et al. (2005). Condición física y tercera edad, valores normales según batería ECFA. Revista área de actividad física I congreso de la asociación española de ciencias del deporte.

20. Oja et al. (1991). A $2 \mathrm{~km}$ walking test for assesing rhb conditions fitness of healthy adults. Int J. Sports Med. 12: $356-362$.

21. Isasi et al. (2003). Physical fitness and c - reactive protein levels in children and young adults. The Columbia University biomarker study. Pediatrics. 111: $332-328 . \operatorname{Pg} 391-405$.

22. Kvaank Elisabeth et al. (2009). Physical fitness and physical activity at ages 13 years as predictor of CVD risk factors: Oslo Youth Study. Pediatrics. 123: e $80-\mathrm{e} 86$.

23. Sánchez, García et al. (2007). Valoración de la condición física general de las selecciones extremeñas de balonmano. Revista Digital deportiva. 3(1), 9-20.

24. Sexton, A. et al. (1993). Value of longitudinal Studies of exercise fitness test. Pediatrics. 1993: 730 - 736 .

25. Beunen, G et al. (1990). Tracking and prediction to various populations. The EUROFIT test to physical fitness. CDDS - CE.

26. Glendhill et al. (2001). Introduction to the review papers pertaining to the components of the canadian physical activity, fitness and life style appraisal. Can. J. Appl. Physiol. 262(2): 157-160.1

27. Meyer et al. (1987). Imagen del yo en los adolescentes y posición del escolar en educación física y deportiva. Infancia y aprendizaje. 37: 45-56.

28. Prat et al. (1987). Batería EUROFIT II. Estandarización y baremación de la batería EUROFIT en base a una muestra en la población catalana. Revista sobre investigación en las ciencias de la educación física y el deporte.

29. Suni et al (1996). Development of health releated fitness test battery for adults: aspects of reliability. Arch. Phys. med. rehab. 73: 399-405. 
30. Laukanen et al. (1996). Development of health releated fitness test battery for adults: aspects of reliability. Arch. Phys. med. rehab. 74: 399 - 405.

31. Miilumpalo et al. (1996). Development of health releated fitness test battery for adults: aspects of reliability. Arch. Phys. med. rehab. 75: 399-405.

32. Passanennet al. (1996). Development of health releated fitness test battery for adults: aspects of reliability. Arch. Phys. med. rehab. 76: 399-405.

33. [32] Vuori et al. (1996). Development of health releated fitness test battery for adults: aspects of reliability. Arch. Phys. med. rehab. 77: $399-405$.

34. Bos et al. (1996). Development of health releated fitness test battery for adults: aspects of reliability. Arch. Phys. med. rehab. 78: 399-405.

35. Gallardo. (1999). Valoración de la Condición Física saludable en adultos (II), aplicabilidad, fiabilidad, y valores normativos de la batería AFISAL - INEFC. Educación Física y Deportes. 54: 54-65.

36. Rodríguez et al. (1999). Valoración de la Condición Física saludable en adultos (II), aplicabilidad, fiabilidad, y valores normativos de la batería AFISAL - INEFC. Educación Física y Deportes. 55: 54-65.

37. Valenzuela et al. (1999). Valoración de la Condición Física saludable en adultos (II), aplicabilidad, fiabilidad, y valores normativos de la batería AFISAL - INEFC. Educación Física y Deportes. 56: 54- 65.

38. Marina et al. (1999). Valoración de la Condición Física saludable en adultos (I), aplicabilidad, fiabilidad, y valores normativos de la batería AFISAL - INEFC. Educación Física y Deportes. 52: 54 - 75.

39. Prwezeda et al. (1990). Report on the implemetation of Eurofit and other analisys of test in assesing physical fitness. CDDS - CE.

40. Sikorsky et al. (1990). Report on the implemetation of eurofit and other analisys of test in assesing physical fitness. CDDS - CE.

41. Lee et al. (1995). Exercise intensity and longevity in men. J. med. Jorn. Asso. 3: 81 - 86 .

42. Brohua et al.( 1993). The step test. A simple method for measuring physical fitness for hard muscular work in adult man. Canadian review of biology $33: 82-87$.
43. Allied dunbar national fitness Survey. (1992). A report on activity patterns and fitness levels. The Sports council and the health education autority.

44. Casajús et al. (1992). Actividad física en el niño escolar. Características antropométricas, composición corporal y madurez. Tesis doctoral. Universidad de Zaragoza.

45. Castillejo et al. (1992). La educación Física para la salud. Propuesta de reconocimiento del pie cavo o plano. Tesis doctoral. Universidad de Andalucia.

46. Capelli et al. (1991). Maximal explosive power and aerobic excersice in humans. Shweisz Zthrz Sportmed. 39: 103-111.

47. Bosco et al. (1988). Valoraciones funcionales de la fuerza dinámica, y de la potencia anaeróbica aláctica. ITFCA.

48. Alvero Cruz et al. (1992). Valoración funcional del deportista mediante la cineantropometría. Estudio en medios fondistas andaluces. Colegio oficial de profesores licenciados en educación física de Andalucía.

49. Cureton et al. (1988). Muscle hypertrophy in men and women. Med. Sci. Sports. Exc.

50. Delgado et al (1997). El entrenamiento de las cualidades físicas en la enseñanza obligatoria. Salud vs rendimiento. Revista de ciencias físicas y del deporte. $\operatorname{Pg} 15-26$.

51. [50] Gallo et al. (1990). Correlatin between $\mathrm{VO}_{2}$ max values obtained indirectly from the two proposed in the EUROFIT test teory for the measurement for cardiovascular resistance. World Congrese of Sports Medicine.

52. Glenmarck et al. (2000). Skeletal muscle fibers types, physical performance, physical activity and attitude to the physical activity in women and men. Acta Physiologyca Scandinavica.

53. Izquierdo et al (1996). Aspectos técnicos condicionantes de la manifestación explosiva de la fuerza. Aplicación a los saltos. Revista española de educación física y deportes.

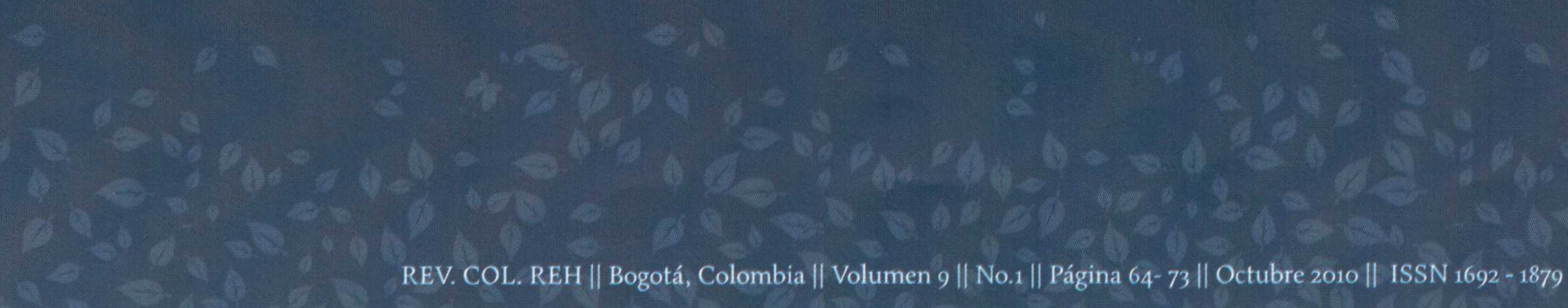

